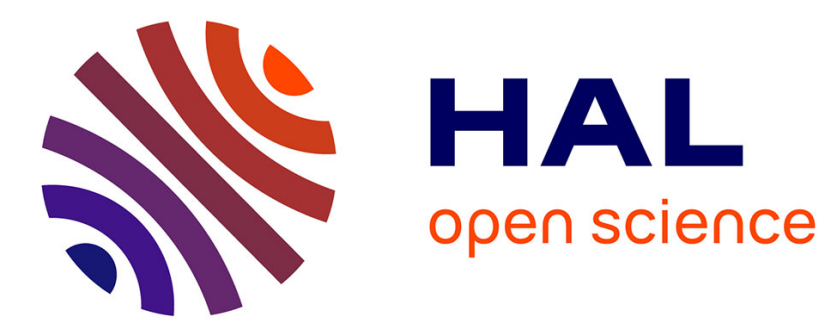

\title{
Genetic algorithm based correlations for heat transfer calculation on concave surfaces
}

\author{
L. Momayez, Pierre Dupont, B Popescu, O. Lottin, H. Peerhossaini
}

\section{To cite this version:}

L. Momayez, Pierre Dupont, B Popescu, O. Lottin, H. Peerhossaini. Genetic algorithm based correlations for heat transfer calculation on concave surfaces. Applied Thermal Engineering, 2009, 29 (17-18), pp.3476. 10.1016/j.applthermaleng.2009.05.025 . hal-00537996

\section{HAL Id: hal-00537996 \\ https://hal.science/hal-00537996}

Submitted on 20 Nov 2010

HAL is a multi-disciplinary open access archive for the deposit and dissemination of scientific research documents, whether they are published or not. The documents may come from teaching and research institutions in France or abroad, or from public or private research centers.
L'archive ouverte pluridisciplinaire HAL, est destinée au dépôt et à la diffusion de documents scientifiques de niveau recherche, publiés ou non, émanant des établissements d'enseignement et de recherche français ou étrangers, des laboratoires publics ou privés. 


\section{Accepted Manuscript}

Genetic algorithm based correlations for heat transfer calculation on concave surfaces

L. Momayez, P. Dupont, B Popescu, O. Lottin, H. Peerhossaini

PII:

S1359-4311(09)00178-1

DOI:

10.1016/j.applthermaleng.2009.05.025

Reference:

ATE 2827

To appear in:

Applied Thermal Engineering

\section{APPLIED \\ THERMAL \\ ENGINEERING}

Received Date: $\quad 12$ July 2008

Revised Date: $\quad 17$ April 2009

Accepted Date: $\quad 31$ May 2009

Please cite this article as: L. Momayez, P. Dupont, B. Popescu, O. Lottin, H. Peerhossaini, Genetic algorithm based correlations for heat transfer calculation on concave surfaces, Applied Thermal Engineering (2009), doi: 10.1016/ j.applthermaleng.2009.05.025

This is a PDF file of an unedited manuscript that has been accepted for publication. As a service to our customers we are providing this early version of the manuscript. The manuscript will undergo copyediting, typesetting, and review of the resulting proof before it is published in its final form. Please note that during the production process errors may be discovered which could affect the content, and all legal disclaimers that apply to the journal pertain. 


\title{
GENETIC ALGORITHM BASED CORRELATIONS FOR HEAT TRANSFER CALCULATION ON CONCAVE SURFACES
}

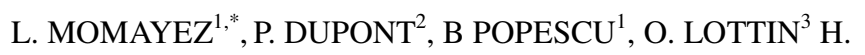 \\ PEERHOSSAINI $^{1 * *}$ \\ ${ }^{1}$ Laboratoire de Thermocinétique, CNRS-UMR 6607, Ecole Polytechnique de \\ l’Université de Nantes, BP 50609, F-44306, Nantes, France \\ *Khorramshahr University of Marine Sciences and Technology, PB 669, 64199- \\ 43175, Iran \\ 2LGCGM, EA3913, INSA de Rennes, Campus Beaulieu, 35043 Rennes cedex, \\ France \\ ${ }^{3}$ Laboratoire d'Energétique et de Mécanique Théorique et Appliquée, LEMTA, 2 \\ avenue de la forêt de Haye, BP 160, 54504 Vandoeuvre les Nancy Cedex, France \\ **Corresponding author: Tel: +33 240683124-40, fax: +33 240683141
} hassan.peerhossaini@univ-nantes.fr

\section{ABSTRACT}

Laboratory experiments were carried out on a boundary layer over a concave wall subject to Görtler instability in the presence of forced wavelengths. Measurements of the local heat transfer along the concave wall permit calculation of the local Stanton number St for various axial positions $x$, nominal velocity $U_{n}$, the wavelength $\lambda$ and strength $d_{w}$ of the forced initial perturbations. The relation among these variables was constructed by a phenomenological argument in the form of classical power law correlations: $S t=a\left(\operatorname{Re}_{\theta}\right)^{b}\left(\frac{x}{R}\right)^{c}\left(\frac{\lambda}{\theta}\right)^{d}$. Correlations constants are then derived by genetic algorithm methods. The calculated Stanton number is in good agreement with the experimental results. In this paper we propose correlations for the calculation of heat transfer on concave surfaces (such as gas 
turbine blade pressure side) where the flow is complex due to the presence of streamwise vortices.

Key words: Görtler instability, concave boundary layer, thermal boundary layer, wall heat transfer, heat transfer intensification, genetic algorithm, correlations, gas turbine blade cooling

\section{NOMENCLATURE}

$C p$
$\mathrm{~d}_{\mathrm{w}}$
$\mathrm{G}_{\theta}$
$H$
$\mathrm{~T}_{\infty}$
$\mathrm{T}_{\mathrm{w}}$
$U$
$\mathrm{U}_{\mathrm{n}}$

$\mathrm{U}_{\mathrm{pw}}$

heat capacity ( $/ \mathrm{kg} . \mathrm{K})$

wire diameter $(\mathrm{m})$

Görtler number $\frac{\mathrm{U}_{\mathrm{n}} \theta}{v} \sqrt{\frac{\theta}{\mathrm{R}}}$

height of the counter wall, $H=0.15$ (m) free-stream temperature $(\mathrm{K})$

wall temperature $(\mathrm{K})$

longitudinal component of velocity $(\mathrm{m} / \mathrm{s})$

Free-stream velocity or nominal velocity $(\mathrm{m} / \mathrm{s})$

potential wall velocity,

$$
\mathrm{U}_{\mathrm{pw}}=\frac{\mathrm{U}_{\mathrm{n}}}{\frac{\mathrm{R}}{\mathrm{H}} \ln \left(1-\frac{\mathrm{H}}{\mathrm{R}}\right)}(\mathrm{m} / \mathrm{s})
$$

$x$

$y$

$z$

$\operatorname{Pr}$

$R$

$R e$

St

$\alpha$

K

v

$\rho$

$\varphi_{\mathrm{w}}$

$\theta$

$N u$ streamwise direction (m)

normal to the wall direction (m)

spanwise direction (m)

Prandtl number, $\mathrm{Pr}=\mathrm{v} / \mathrm{k}$

wall radius of curvature $(\mathrm{m})$

Reynolds number $\mathrm{U}_{\mathrm{n}} \mathrm{x} / \mathrm{v}$

Stanton number

$\varphi_{\mathrm{w}} / \rho \mathrm{C}_{\mathrm{p}} \mathrm{U}_{\mathrm{pw}}\left(\mathrm{T}_{\mathrm{wall}}-\mathrm{T}_{\infty}\right)$

wave number of initial perturbations:

$\alpha=2 \pi / \lambda$

thermal diffusivity $\left(\mathrm{m}^{2} / \mathrm{s}\right)$

kinematic viscosity $\left(\mathrm{m}^{2} / \mathrm{s}\right)$

density $(\mathrm{kg} / \mathrm{m} 3)$

wall heat flux $\left(\mathrm{w} / \mathrm{m}^{2}\right)$

Blasius momentum thickness

$\theta=0.664 x(\operatorname{Re})^{-1 / 2}$

Local Nusselt number $\mathrm{Nu}=\varphi_{\mathrm{p}} \mathrm{x} / \lambda \Delta \theta$ 


\section{INTRODUCTION}

Boundary layers with concave streamlines arise in several situations of practical importance such as the pressure side of gas turbine blade, combustion chamber, diffusers etc. In laminar regimes such boundary layers are susceptible to hydrodynamic centrifugal instability, generally called Görtler instability after the original work of Görtler [1]. At the start of the instability, the boundary layer is still a laminar boundary layer over which an array of streamwise vortices (called Görtler vortices) is superposed, as is shown on figure 1. Görtler vortices in their nonlinear stage of development drastically modify momentum and heat transfer on the wall.

The control parameter $G_{\theta}$, called the Görtler number, is defined as $\mathrm{G}_{\theta}=\frac{\mathrm{U}_{\infty} \theta}{v}\left(\frac{\theta}{\mathrm{R}}\right)^{1 / 2}$, where $U_{\infty}, \theta$, and $\mathrm{R}$ are freestream velocity, momentum thickness and radius of curvature of the concave wall respectively, and $v$ is the kinematic viscosity of the fluid.

The Görtler number can be recast as $G_{\theta}=\Lambda_{\lambda}(\alpha \theta)^{3 / 2}$, where $\Lambda_{\lambda}$ is the wavelength parameter, defined as $\Lambda_{\lambda}=\frac{U_{\infty} R}{v}\left(\frac{\lambda}{R}\right)^{3 / 2}$, and $\lambda$ the vortex wavelength $\left(\lambda=\frac{2 \pi}{\alpha}\right)$. The parameter $\Lambda_{\lambda}$ is constant in an experiment. with a given wavelength.

Centrifugal instabilities have a great influence on the heat transfer over curved surfaces such as gas turbine blades [2]\&[3]. The heat transfer is enhanced over a concave surface while it is inhibited over a convex wall. In the special case of gas turbine blades Görtler vortices play an important role. In fact, for calculation of thermal stresses in the turbine blade one needs to know the amount of heat transferred from the combustion products to the blade. Heat transfer correlations existing in the open literature do not take into account the extra thermal load caused by the streamwise vortices. Therefore, they underestimate the convective heat transfer coefficient which is used as a boundary condition for the calculation of blade thermal stress loading. This coefficient is also crucial for the design of blade 
internal cooling. In the blade film cooling Görtler vortices can be harmful. Duchêne[4] showed that the Görtler vortices exist on the pressure side of the gas turbine blades and strongly interact with the longitudinal vortices generated by the film cooling jets. In this special case he demonstrated that the Görtler instability is responsible for a slight reduction in the cooling efficiency.

In the present work we measure detailed heat transfer rates for different Görtler vortex wavelengths and two upstream perturbation strengths. The initial disturbances are generated by means of fixed manipulation grids made up of a series of forcing wires of diameter $0.18 \mathrm{~mm}$ or $0.80 \mathrm{~mm}$ and with $5,10,20,30,40,50$, and $60 \mathrm{~mm}$ spanwise wavelengths. The grid is placed vertically $4 \mathrm{~mm}$ upstream of the leading edge of the concave wall. The principal role of the perturbation (manipulator) grid is to fix the distance between vortices and their spanwise positions. The concave wall is heated with a constant heat flux $\varphi_{p}=200 \mathrm{~W} / \mathrm{m}^{2}$ using double-layered resistive sheets. Wall temperature was measured with one hundred and ninety chromel-alumel-type thermocouples of $80 \mu \mathrm{m}$ diameter embedded in the wall. Thermocouples are placed in such a way that the longitudinal evolution of the wall temperature can be followed as well as its spanwise variation at several longitudinal positions.

The vortex wavelength of the measurements carried out in this work covers a very large range in $\Lambda_{\lambda}$, between 58 to 3841 , corresponding to regions in the damped zone of the linear theory, passing through the most amplified Görtler vortex $(\Lambda \approx 210)$ and extending into the weak amplification $(\Lambda \approx 3841)$ zone. Data used in the present work was obtained from more than 44 experimental configurations with constant wall radius of curvature.

In previous work, Momayez et al. [5] have demonstrated the difficulties in reducing the number of governing parameters for heat transfer on a concave wall. Those complex situations have not been expressed in terms of correlations. The problem arises either from the number of parameters (4) or from the unknown mechanisms that influence the heat transfer. 
In this paper we derive and optimize correlations for the convective heat transfer coefficient in a boundary layer over a concave wall by using Genetic Algorithm (GA) methods.

\section{EXPERIMENTAL SET UP AND METHODS}

Experiments were carried out in the boundary layer on a concave-convex model mounted in a laminar open-loop wind tunnel described in [5]. The nominal freestream velocity could vary between 1.5 and $10 \mathrm{~m} / \mathrm{s}$ with constant turbulence intensity of $0.7 \%$.

The concave-convex model (Figure 2) has four main parts:

- the leading edge in the shape of a thick laminar airfoil (NACA-0025)

- the concave part (radius of curvature $65 \mathrm{~cm}$ ) in which the measurements were

made

- the convex part (radius of curvature $15 \mathrm{~cm}$ )

- the trailing edge, a flat plate that can rotate around the center of curvature of the convex section

In order to minimize the longitudinal pressure gradient, a Plexiglass counter wall (opposite to the concave wall) was fixed on top of the model. The origin of the curvilinear axial coordinate $\mathrm{x}$ is fixed at the leading edge and the concave wall starts at $\mathrm{x}=9 \mathrm{~cm}$. Görtler vortices are generated as the result of the amplification by the centrifugal instability of upstream perturbations entering the concave boundary layer. In this study the spanwise position of the Görtler vortices was fixed by forcing predetermined wavelengths upstream of the leading edge. This was done by a manipulation (perturbation) grid made of variable-diameter wires with different wavelengths (spanwise distances $\lambda$ ) placed vertically $4 \mathrm{~mm}$ upstream of the leading edge. In order to analyze the response of the boundary layer to the upstream perturbations, we study the reference case $(\lambda=\infty$, no forcing) and then the cases corresponding to increasing numbers of wires: $\lambda=6,5,4,3,2,1$ and $0.5 \mathrm{~cm}$, with a small wire diameter of $\mathrm{d}_{\mathrm{w}}=0.18 \mathrm{~mm}$ (weak perturbations) and a large wire diameter $\mathrm{d}_{\mathrm{w}}=0.8 \mathrm{~mm}$ (strong perturbation). 
The model surface was covered with a thin $(130 \mu \mathrm{m})$ resistance film made of a $70 \mu \mathrm{m}$ constantan layer glued to a $60 \mu \mathrm{m}$ Kapton film and was heated by the Joule effect. 196 chromel-alumel thermocouples of $80 \mu \mathrm{m}$ bead measured the temperature on the Kapton side of the film. To reduce heat loss from the back of the model wall, it was insulated with phenolic foam $(\mathrm{k}=0.02 \mathrm{~W} / \mathrm{mK})$ in which eight thermocouples were implanted. Measurements showed that heat losses by conduction from the back of the model were 6 to $8 \mathrm{~W} / \mathrm{m}^{2}$, that is, between 3 and $4 \%$ of the imposed heat flux on the wall. Thermocouples were used to measure the wall temperature along three streamwise paths and several spanwise lines. Fluid temperature in the boundary layer was measured by a thermocouple probe similar to that used by Blackwelder and Moffat (Blackwelder and Moffat [6]). Experiments were carried out at $\mathrm{U}_{\mathrm{n}}=2$, $3,4.8,7 \mathrm{~m} / \mathrm{s}$ and the wall heat flux was $\varphi=200 \mathrm{~W} / \mathrm{m}^{2}$. The nominal flow velocity out of the boundary layer was measured upstream of the model's leading edge by a Pitot tube. In the present experiments $\mathrm{U}_{\mathrm{pw}} \approx 0.88 \mathrm{U}_{\mathrm{n}}$ is obtained theoretically by calculating the potential velocity $\mathrm{U}_{\mathrm{p}}(\mathrm{y})$ at $\mathrm{y}=0$ (Momayez [7]).

\section{EFFECTS OF GÖRTLER INSTABILITY ON THE WALL HEAT} TRANSFER

The cross-sectional heat advection brought about by Görtler vortices in an unstable boundary layer over a concave wall makes the heat transfer very different from that in a plain laminar boundary layer. Görtler vortices at their onset are very weak (Floryan [8], Peerhossaini and Wesferid [9]). According to the linear theory, the boundary layer becomes unstable at some critical Görtler number (for instance, at $\mathrm{G}_{\theta}=0.46$ in Floryan and Saric [10]), and its linear amplification (half of its growth in terms of axial distance) occurs before any disturbance can be detected. The first effect of Görtler vortices on wall heat transfer thus appears in the nonlinear state of the vortices. This fact is evident in Figure 3, which plots Stanton number versus Reynolds number for five nominal velocities. This figure shows the results for the reference case of $U_{n}=3 \mathrm{~m} / \mathrm{s}$ without a vortex-triggering grid. The results are 
also compared with Blasius heat transfer correlation for a laminar boundary layer (laminar flat-plate) and the correlation for a turbulent boundary layer on a flat plate (Kays and Crawford [11]) with constant wall heat flux.

On Figure 3 we have shown the evolution of the Stanton number as a function of Reynolds number for $\mathrm{U}_{\mathrm{n}}=3 \mathrm{~m} / \mathrm{s}$ in the absence of the vortex-triggering grid (for simplicity). Note that in this Figure the experimental points follow the laminar flat-plate curve up to certain Reynolds numbers, confirming the initial very weak or zero effect of curvature on heat transfer. The measurements then diverge from the laminar flat-plate curve and after a constant-Stanton-number "plateau", heat transfer increases drastically before being saturated by turbulence. On Figure 3 laminar flat plate (Blasius) and turbulent flat plate boundary layer curves are used as reference for the evolution of heat transfer along the concave surface.

Four different stages in the longitudinal evolution of the boundary layer can be distinguished in Figure4:

- $\quad$ zone OA: this zone corresponds to the leading edge and its junction with the concave part. In this zone St follows approximately the curves corresponding to the laminar boundary layer on the flat plate (2D steady-laminar flow). Deviation of the first few points is due to flow acceleration at the leading edge.

- zone $\mathrm{AB}$ : here heat transfer on the concave wall deviates gradually from the flat plate by the appearance of a "plateau" with roughly constant Stanton number. This heat-transfer intensification is related to the growth of the Görtler vortices under the effect of centrifugal instability (3D steady-laminar flow).

zone $\mathrm{BC}$ : the heat transfer coefficient gradually reaches values close to or above turbulent boundary layer values on a flat plate. This rapid increase in heat transfer coefficient (St) is due to the secondary instability of the Görtler vortices (Toé et al. [12]). Indeed, once the Görtler vortices reach sufficient strength, subsequent longitudinal velocity gradients cause inflection points where shear instability develops (3D unsteady flow); this secondary instability grows rapidly and induces a premature (compared to the flat plate) boundary-layer transition to turbulence. 
- zone $\mathrm{CD}$ : heat transfer ceases to increase and follows the flat-plate turbulent curve. It has been observed (Ajakh et al. [13]) that turbulent spots are present in the boundary layer at these Görtler numbers. As Kestoras and Simon [14] and also Toé et al. [15] have noted, the heat-transfer level of the turbulent boundary layer is higher on the concave wall than on a flat plate. This heat-transfer enhancement in the turbulent regime can be explained by the mechanisms responsible for heattransfer enhancement in the laminar flow: the primary Görtler instability induces longitudinal vortices that are not completely broken down by transition and thus are still weakly present. The secondary instability induces large-scale coherent vortices in the turbulent flow that are more efficient for transport than the smaller fine grain turbulent scales.

An attempt to correlate the variations of Stanton number with Görtler or Reynolds number has not given a conclusive result. In fact, Momayez et al.[5] plotted experimentally obtained values of the Stanton number versus Reynolds and Görtler number as shown on Figures 3 and 5. Instead of collapsing together the curves show a certain dispersion, implying that probably neither Gr nor Re is the proper control parameter of the problem. Question rises as to whether an other combination of the problem variables $\left(\mathrm{U}_{\mathrm{n}}, \lambda, \mathrm{R}, \mathrm{X}, \mathrm{)}\right.$ can better correlate the results. For this purpose and given the very large number of the data collected in 44 series of measurement we have used the Genetic Algorithm method to search such correlations.

\section{GENETIC ALGORITHM}

Genetic algorithm is a branch of evolutionary computing, a swiftly growing area of reproduction intelligence, which is a search-and-optimization technique based on natural selection. Genetic algorithms (GAs) are motivated by Darwin's theory of evolution. Simply put, problems are solved by an evolutionary process that yields a best (fittest) solution (survivor) — in other words, the solution evolves. The GA is a computer simulation of such evolution in which the user provides the environment (function) in which the population must evolve. Evolutionary computing was introduced in the 1960s by Rechenberg in his book 
Evolution strategies ${ }^{1}$ and was later developed by other researchers. GAs were created by John Holland [16] and developed by him, his students and colleagues, culminating in Holland's 1975 book, Adaptation in Natural and Artificial Systems. In 1992 John Koza [17][18][19] used GA to develop programs to carry out certain tasks, calling his method 'genetic programming' (GP). If we are solving a problem, we are usually looking for a solution that is better than all others. The space of all reasonable solutions (the set of solutions among which the desired solution resides) is called the search space (also state space). Each point in the search space represents one possible solution. Each possible solution is distinguished by its value (or fitness) for the problem. With GA we look for the best solution, represented by one point in the search space, among a number of possible solutions. Looking for a solution is then equivalent to looking for some extreme value (minimum or maximum) in the search space. At times the search space may be well defined, but usually only a few points in it are known. The process of finding solutions in GA generates other points (possible solutions) as evolution proceeds.

In the field of acoustics, Sato et al. [20] used GA to design a concert hall with optimal acoustic properties, maximizing the sound quality for the audience, the conductor, and the on-stage musicians. Porto et al. [21] used evolutionary programming to train neural networks to distinguish between sonar reflections from different types of objects: man-made metal spheres, seamounts, fish and plant life, and random background noise. Tang et al. [22] survey the uses of genetic algorithms in acoustics and signal processing.

In aerospace engineering, Obayashi et al. [23] used a multiple-objective GA to design the wing shape for a supersonic aircraft. Williams et al [24] applied genetic algorithms to spacing satellite orbits in order to minimize coverage black-outs. Keane and Brown [25] used a GA to design a load-bearing truss or boom to be assembled in orbit and used for satellites, space stations and other aerospace construction projects.

\footnotetext{
${ }^{1}$ Rechenberg, I., / Evolutionsstrategie: Optimierung technischer Systeme und Prinzipien der biologischen Evolution /, FrommannHolzboog, Stuttgart, 1973
} 
The GA used in this study is written in C. A MATLAB ${ }^{\circledR}$.Implementation of the algorithm in MATLAB simplifies its use and also gives access (from the command line) to some of its main parameters in order to increase performance in particular cases. It is designed to minimize/maximize complex functions and is based on real number encoding. It can handle various characteristics (discontinuity, multimodality, and the like) without being optimized for a particular type of function and returns good results with the most common benchmark functions and with new ones as well [25] [27] [28]. The algorithm integrates classic and new features, such as the ability to shape the sampling bias and a new sampling type for the BLX- $\alpha$ crossover operator [29][30][31][32]. We recommend this GA for solving problems on which traditional methods fail to return the global minimum/maximum and especially when the function characteristics are not known.

\subsection{CHOICE OF THE CONTROL PARAMETER}

In a first correlation among the different problem parameters we assume that the wall temperature depends on $U_{\infty}, x, \lambda, R$, that is: $\mathrm{T}\left(\mathrm{U}_{\infty}, \mathrm{x}, \lambda, \mathrm{R}\right)$. In classical dimensionless heat transfer form it can be reduced toSt $\left(G_{\theta}, \Lambda, \operatorname{Re}_{x}\right)$, where $G_{\theta}$ (Görtler number based on momentum thickness) is the principal parameter and $\Lambda$ is the non-dimensional wavelength in the Görtler instability. For this case we assume the correlation law $\mathrm{St}=\mathrm{aG}_{\theta}^{\mathrm{b}} \Lambda^{\mathrm{c}} \operatorname{Re}_{\mathrm{x}}^{\mathrm{d}}$, where $\mathrm{a}, \mathrm{b}, \mathrm{c}, \mathrm{d}$ are the unknown coefficients determined by the GA program. The coefficients are calculated separately for each zone $(\mathrm{OA}, \mathrm{AB}, \mathrm{BC}$ and $\mathrm{CD})$. Table 2 presents the values of mean error $(\%)$ for this correlation calculated for different nominal velocities $U_{n}$ and perturbation strength (wire diameter).

In a second try for correlation we consider the influence of the curvature through the dimensionless number $\mathrm{x} / \mathrm{R}$, which represents the local angle of rotation of flow streamlines. This choice gives more weight to the radius of curvature of the concave wall. The influence of $U_{n}$ is still taken into account by the 
Reynolds number or the Görtler number, and we naturally nondimensionalize the wavelength with the boundary-layer thickness. Then the expected relation becomes:

$$
S t=a\left(G_{\theta} \sqrt{\frac{R}{\theta}}\right)^{b}\left(\frac{x}{R}\right)^{c}\left(\frac{\lambda}{\theta}\right)^{d}=a\left(\operatorname{Re}_{\theta}\right)^{b}\left(\frac{x}{R}\right)^{c}\left(\frac{\lambda}{\theta}\right)^{d}
$$

Coefficients a, b, c, and d are calculated for different zones (OA, AB, BC, CD) and shown in Table 1 (to save space we have shown the coefficients only for this correlation which, as we see later, fits the best the experimental results). Values of mean errors are shown in Table 2. In the third try for correlation we attempted to take into account all dimensional parameters in a minimum number of dimensionless numbers: $\mathrm{St}=\mathrm{a}\left(\frac{\mathrm{U}_{\mathrm{n}} \lambda}{\mathrm{v}}\right)^{\mathrm{b}}\left(\frac{\theta}{\mathrm{R}}\right)^{\mathrm{c}}$. Again we have calculated by GA the values of $\mathrm{a}, \mathrm{b}$, c, for the different zones; the mean errors are shown in Table 2.

\subsection{RESULTS}

Before presenting the results we recall that the range of parameters covered in the experiments and in simulations reported in this work is given in Table 3.

From the construction point of view, the third correlation is obviously the worst fit, clearly because the hypothesized law lacks enough parameters to follow the complex experimental results. The two other correlations both have four control parameters and give roughly the same uncertainty. The final decision was made by putting in the three correlations the (flow and geometrical) parameters corresponding to 44 experimental configurations and comparing the Stanton-number versus Görtler-number curves obtained from the correlations with the corresponding experimental curves. The best results were obtained with the second correlation, where the effect of axial distance, relative curvature and relative wavelength are explicitly taken into account. Figures 6 and 7 compare the results of the second correlation with the corresponding experimental results. We have chosen only a few cases among the 44 optimization cases and are compared with 44 corresponding experimental cases.

In Table 1 the relative magnitudes of coefficients (b, $c$ and $d$ ) reveal the sensitivity of the heat transfer to the Reynolds number, curvature and wavelength 
respectively. In zone OA, b is larger than $\mathrm{c}$ and $\mathrm{d}$, which is consistent with a nearly 2D steady laminar flow. Otherwise, in zone $\mathrm{AB}, \mathrm{c}$ is larger than $\mathrm{b}$ and $\mathrm{d}$, implying that curvature is the dominant mechanism of heat transfer sensitivity. This is in agreement with a strong 3D steady laminar flow which dominates in this zone. In zone $\mathrm{BC}$, we again have a strong Reynolds-number effect with sensitivity to the wavelength. This result is in agreement with a $3 \mathrm{D}$ unsteady flow with transition governed by the secondary Görtler instability. Finally in zone CD, the dominant parameter is the Reynolds number with sensitivity to curvature since in this zone the flow is fully turbulent therefore, the organized vortex structure is already disappeared and the notion of wavelength has lost its significance. However, the centrifugal effects due to the curvature are still active.

Zones in which each set of constants is valid are defined in terms of the value of the non-dimensional Görtler number. Therefore, in applying the correlations these sets of constants should also be valid in the same range of Görtler number defined based on the parameters of the problem in hand..

\section{CONCLUSIONS}

Heat transfer enhancement by streamwise Görtler vortices is considered. Passive flow manipulation for heat transfer intensification is due to the centrifugal instability caused by wall curvature. Previous results of the authors show however, that this heat transfer enhancement is sensitive to the wavelength and strength of the Görtler vortices. Therefore, perturbation grids were fixed upstream of the concave wall to manipulate and to force the wavelength of the vortices. Following a parametric study a large number of experimental data (in the form of the Stanton number as a function of the different flow and geometrical parameters of the problem) is now available. However, it has not been possible to establish a correlation between these parameters which fits the experimental data properly. Searching for such optimal correlation is a nonlinear optimization problem. There exit many methods for this optimization, we have opted for Genetic Algorithm. It can rapidly give sufficiently satisfactory solutions. 
By a phenomenological procedure three possible correlations were submitted to the GA estimator used in search for global optimal estimation of the correlation coefficients. Given the very distinct behavior of the Stanton number in a large range of the Görtler number (which covers 2D laminar, 3D laminar, transitional and turbulent regimes) optimization and evaluation of the mean values of errors were carried out for each zone separately. The results of the correlations were then compared with experimental results. This optimization was also performed for two values of perturbation strength. It was concluded that the most optimal correlation is in the form of $S t=a\left(R_{\theta}\right)^{b}(x / R)^{c}(\lambda / \theta)^{d}$, with values of constant coefficients as given in table 1. 


\section{REFERENCES}

[1] H. Görtler, "On the three-dimensional instability of laminar boundary layers on concave walls." Nacher. Ges. Wiss. Göttingen 2, (NACA TM 1375), (1954), 1-26.

[2]Simon T.W., R. J. Moffat, J.P. Johnston, and W. M. Kays: Report HMT-32, Thermosciences Division, Department of Mechanical Engineering, Stanford University, November 1980.

[3] H. Peerhossaini, "On the effects of streamwise vortices on wall heat transfer", Compact heat exchangers for process industries, ed. R. Shah Begell House Publishers, New York, 1997, 571-589.

[4] Christophe Duchêne, "Etude numérique du refroidissement par film: phénomènes de base et application à la protection thermique des aubes de turbines. Ph.D. dissertation, university of Nantes, France, 1995.

[5] L. Momayez, P. Dupont, H. Peerhossaini, "Effects of vortex organization on heat transfer enhancement by Görtler instability", Int. J. Therm. Sciences, 43, (2004), 753-760.

[6] B.F. Blackwelder, R.J. Moffat, "Design and construction of a low-velocity boundary layer temperature probe." ASME Journal of Heat Transfer, May 1975.

[7] Momayez, L., Dupont, P., Peerhossaini, H., 2004 a , Some unexpected effects of wavelength and perturbation strength on heat transfer enhancement by Görtler instability. Int. J. of Heat and Mass Transfer, 47, 2004. 3783-3795.

[8] J.M. Floryan,, "On the Görtler instability of boundary layers," Prog. Aerospace Sci., Vol. 28, (1991), 235-271.

[9] H. Peerhossaini, J.E. Wesfreid, “On the inner structure of Görtler vortices.” Int. J. Heat Fluid Flow 9, (1988), 12-18.

[10] J.M. Floryan, W.S Saric, "Stability of Görtler vortices in boundary layers", AIAA J. 20, (1982), 316-324.

[11] W.M. Kays, M.E. Crawford, "Convection, heat and mass transfer." Third edition, McGraw-Hill, New York 1993. 
[12] R. Toé, «Etude expérimentale de l'instabilité de Görtler : Instabilité secondaire et effects des tourbillons de Görtler sur les phénomènes de transfer thermique », Ph.D. dissertation, university of Nantes, France, 1999.

[13] A. Ajakh, M.D. Kestoras, R. Toé, H. Peerhossaini , "Influence of forced perturbations in the stagnation region on Görtler instability”, AIAA J. 37 (12), (1999), 1572-77.

[14] M.D. Kestoras, T.W Simon, "Combined effects of concave curvature and high free-stream turbulence intensity on boundary layer heat momentum transport", ASME Winter Annual Meeting, New Orleans, LA, 1993.

[15] R. Toé, A. Ajakh, H. Peerhossaini, "Heat transfer enhancement by Görtler instability", Int. J. Heat and Fluid Flow 23, (2002) 194-204.

[16] J. Holland, “Genetic algorithms.” Scientific American, (July 1992), 66-72.

[17] J. Koza, Forest Bennett, David Andre and Martin Keane, "Genetic Programming” III: Darwinian Invention and Problem Solving. Morgan Kaufmann Publishers, 1999.

[18] J. Koza, M. Keane, M. Streeter, W. Mydlowec, J. Yu and G. Lanza, Genetic Programming IV: Routine Human-Competitive Machine Intelligence. Kluwer Academic Publishers, 2003.

[19] J. Koza, M. Keane and M. Streeter, "Evolving inventions." Scientific American, (2003) $52-59$.

[20] S. Sato, K. Otori, A. Takizawa, H. Sakai, Y. Ando and H. Kawamura, “Applying genetic algorithms to the optimum design of a concert hall." Journal of Sound and Vibration, vol.258, no. 3, (2002), 517-526.

[21] V. Porto, D. Fogel and L. Fogel , "Alternative neural network training methods." IEEE Expert, 10, (1995), 16-22.

[22] K.S. Tang, K.F. Man, S. Kwong and Q. He, "Genetic algorithms and their applications.” IEEE Signal Processing Magazine,13, (1996), 22-37.

[23] Obayashi, Shigeru, Daisuke Sasaki, Yukihiro Takeguchi, and Naoki Hirose, , "Multiobjective evolutionary computation for supersonic wing-shape optimization." IEEE Transactions on Evolutionary Computation, 4, ( 2000), 182-187. 
[24] E. Williams, W. Crossley and T. Lang, "Average and maximum revisit time trade studies for satellite constellations using a multiobjective genetic algorithm." Journal of the Astronautical Sciences, 49, (2001), 385-400.

[25] A.J. Keane, and S.M. Brown, "The design of a satellite boom with enhanced vibration performance using genetic algorithm techniques." In Adaptive Computing in Engineering Design and Control 96 - Proceedings of the Second International Conference, University of Plymouth, .I.C. Parmee (ed), (1999), 107-113.

[26] Z.L. Yang, G.-R. Liu, A modified micro genetic algorithm and inverse identification of material properties of a printed circuit board, In Proceedings of the 4th International Conference on Inverse Problems in Engineering, Rio de Janeiro, Brazil, 2002.

[27] D. Whitley, Rana, K. S Mathias, J. Dzubera, , "Evaluating evolutionary algorithms", Artificial Intelligence, 85, (1996), 245-276.

[28] Z. Michaelewicz, Genetic Algorithms + Data Structures = Evolution Programs, Springer-Verlag, Berlin, 1992.

[29] S. Tsutsui, Multi-parent recombination in genetic algorithms with search space boundary extension by mirroring. In Proceedings of the Fifth International Conference on Parallel Problem Solving from Nature, Springer, Berlin, (1998), $428-437$

[30] S. Tsutsui, M. Yamamura, T. Higuchi, , Multi-parent recombination with simplex crossover in real coded genetic algorithms. In Proceedings of the Genetic and Evolutionary Computation Conference GECCO-99, (1999), 657-664.

[31] S. Tsutsui, "Sampling bias and the search space boundary extension in real coded genetic algorithms." In Proceedings of the Genetic and Evolutionary Computation Conference GECCO-2000, Morgan-Kaufmann, (2000), 211-218.

[32] S. Tsutsui, D. E. Goldberg, Search space boundary extension method in realcoded genetic algorithms, Information Sciences , 133, (2001), 229-247. 


\section{FIGURE CAPTIONS}

Figure 1: Görtler vortices in the boundary layer at $x=490 \mathrm{~mm}$ from the leading edge $\left(U_{n}=2 \mathrm{~m} / \mathrm{s}\right)$.

Figure 2. Schematic diagram of the concave-convex model.

Figure 3. Evolution of the Stanton number as a function of Reynolds number for various free-stream velocities in the absence of the vortex triggering grid

Figure 4. Evolution of Stanton number versus Reynolds number for $U_{n}=3 \mathrm{~m} / \mathrm{s}$ in the absence of the vortex-triggering grid.

Figure 5. Evolution of the Stanton number as a function of Görtler number for various free-stream velocities in the absence of the vortex triggering grid.

Figure 6. Comparison between experimental (filled) and simulated (empty) evolution of Stanton number versus Görtler number for different $\lambda$ and $U_{n}=3 \mathrm{~m} / \mathrm{s}, d_{w}=0.18 \mathrm{~mm}$. Simulation results are obtained from the second correlation.

Figure 7 Comparison between experimental (filled) and simulated (empty) evolution of Stanton number versus Görtler number for different $U$ and $\lambda=30 \mathrm{~mm}, \mathrm{~d}_{\mathrm{w}}=0.18 \mathrm{~mm}$. Simulation results are obtained from the second correlation. 


\section{LIST OF TABLES}

Table 1: Values of different coefficients for second correlation: $S t=a\left(R_{\theta}\right)^{b}(x / R)^{c}(\lambda / \theta)^{d}$

Table 2: Mean values of errors (\%) for different experimental configurations in the present work, for first correlation $S t=\mathrm{aG}_{\theta}^{\mathrm{b}} \Lambda^{\mathrm{c}} \mathrm{Re}_{\mathrm{x}}^{\mathrm{d}}$, for second correlation $\mathrm{St}=\mathrm{a}\left(\mathrm{R}_{\theta}\right)^{\mathrm{b}}(\mathrm{x} / \mathrm{R})^{\mathrm{c}}(\lambda / \theta)^{\mathrm{d}}$, and for third correlation $\mathrm{St}=\mathrm{a}\left(\frac{\mathrm{U}_{\mathrm{n}} \lambda}{v}\right)^{\mathrm{b}}\left(\frac{\theta}{\mathrm{R}}\right)^{\mathrm{c}}$, (a) $\mathrm{d}_{\mathrm{w}}=0.18 \mathrm{~mm}$, (b) $\mathrm{d}_{\mathrm{w}}=0.80 \mathrm{~mm}$ 

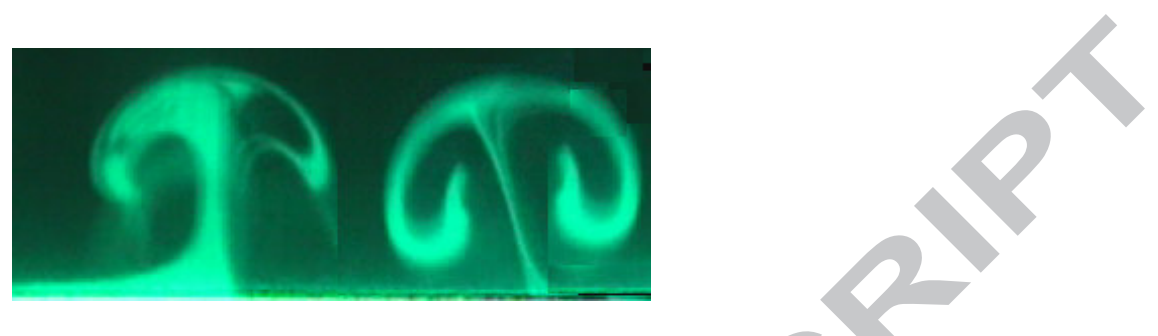

Figure 2: Görtler vortices in the boundary layer at $\mathrm{x}=490 \mathrm{~mm}$ from the leading edge $\left(U_{n}=2 \mathrm{~m} / \mathrm{s}\right)$. 


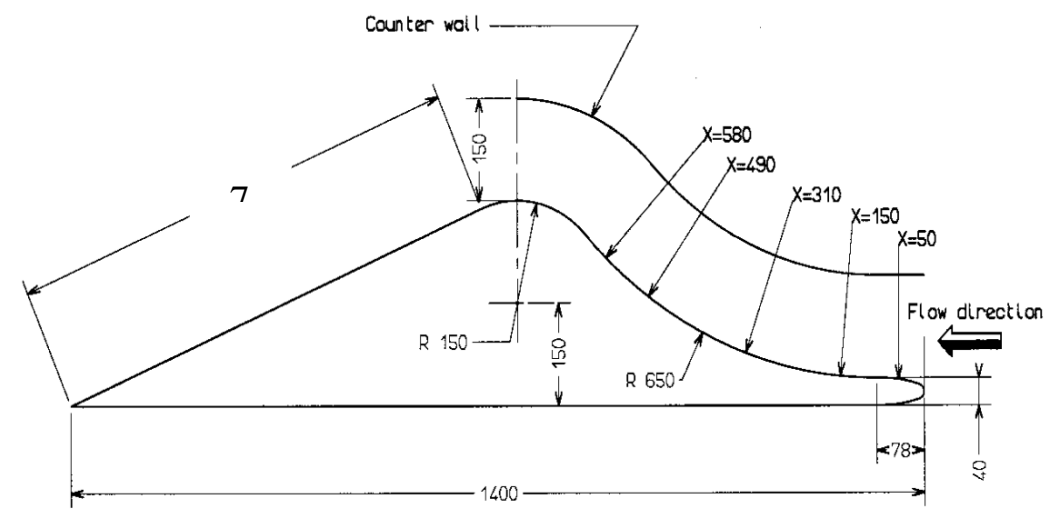

Figure 2. Schematic diagram of the concave-convex model 


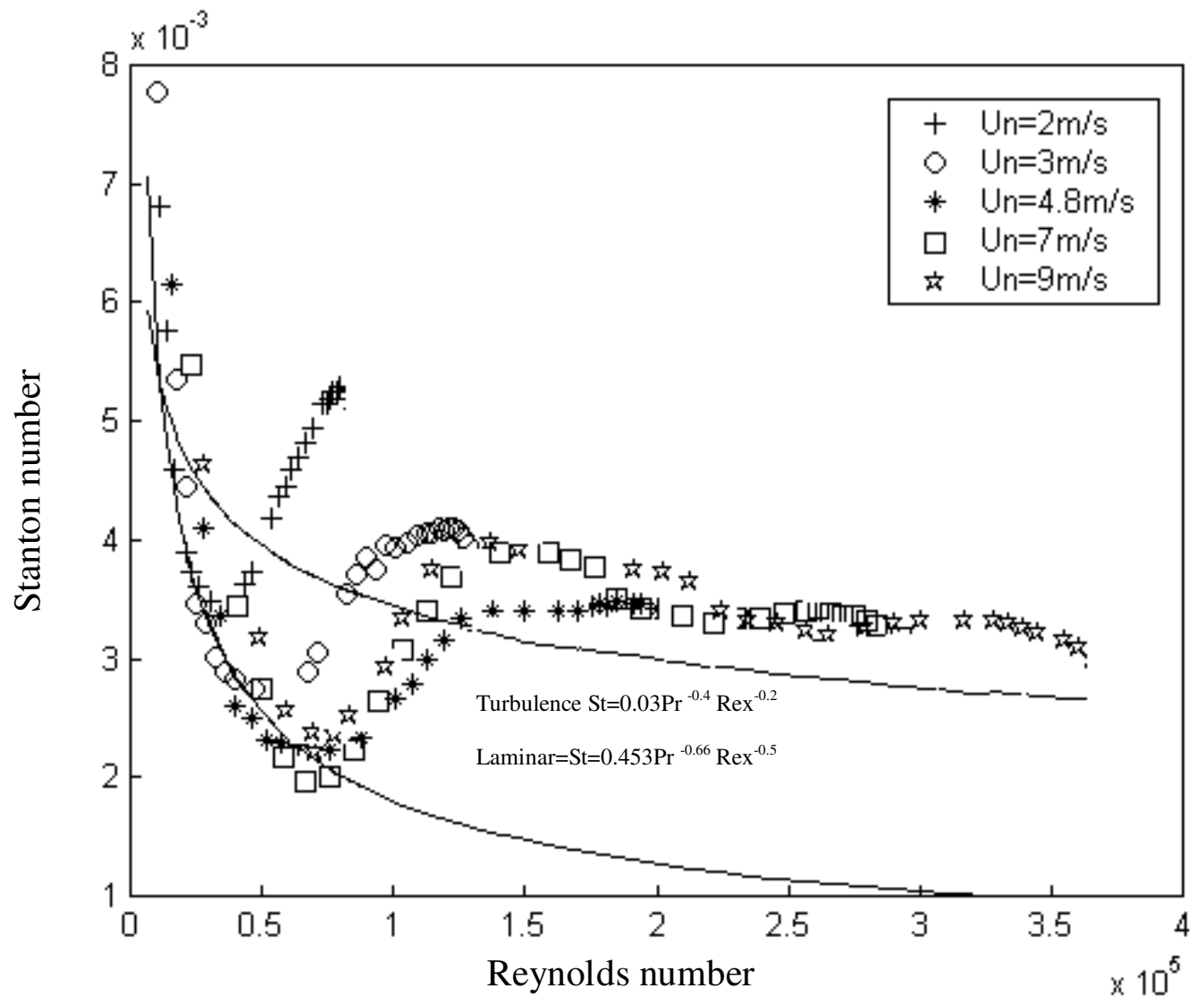

Figure 3. Evolution of the Stanton number as a function of Reynolds number for various free-stream velocities in the absence of the vortex triggering grid 


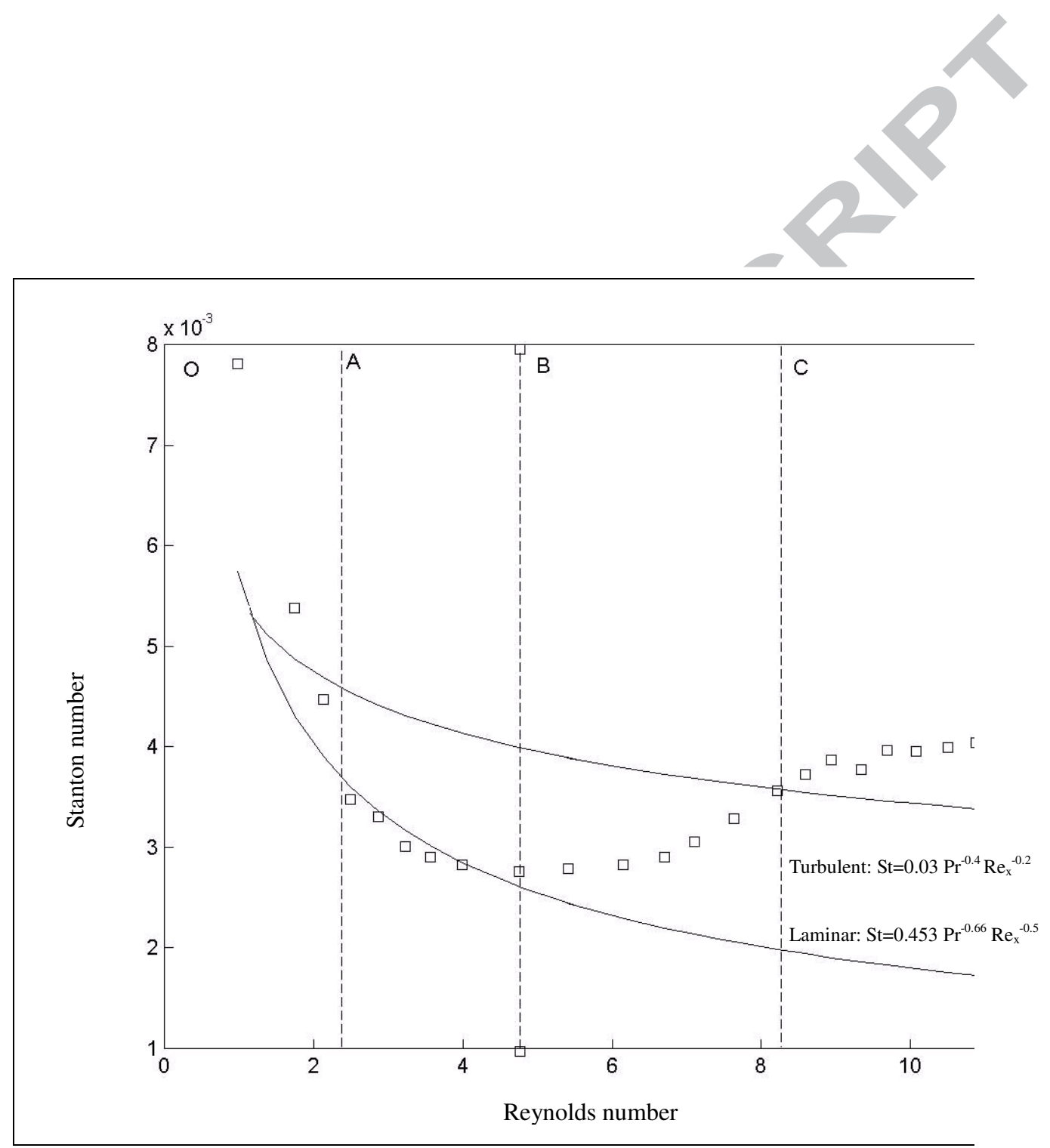

Figure 4. Evolution of Stanton number versus Reynolds number for $\mathrm{U}_{\mathrm{n}}=3 \mathrm{~m} / \mathrm{s}$ in the absence of the vortex-triggering grid. 


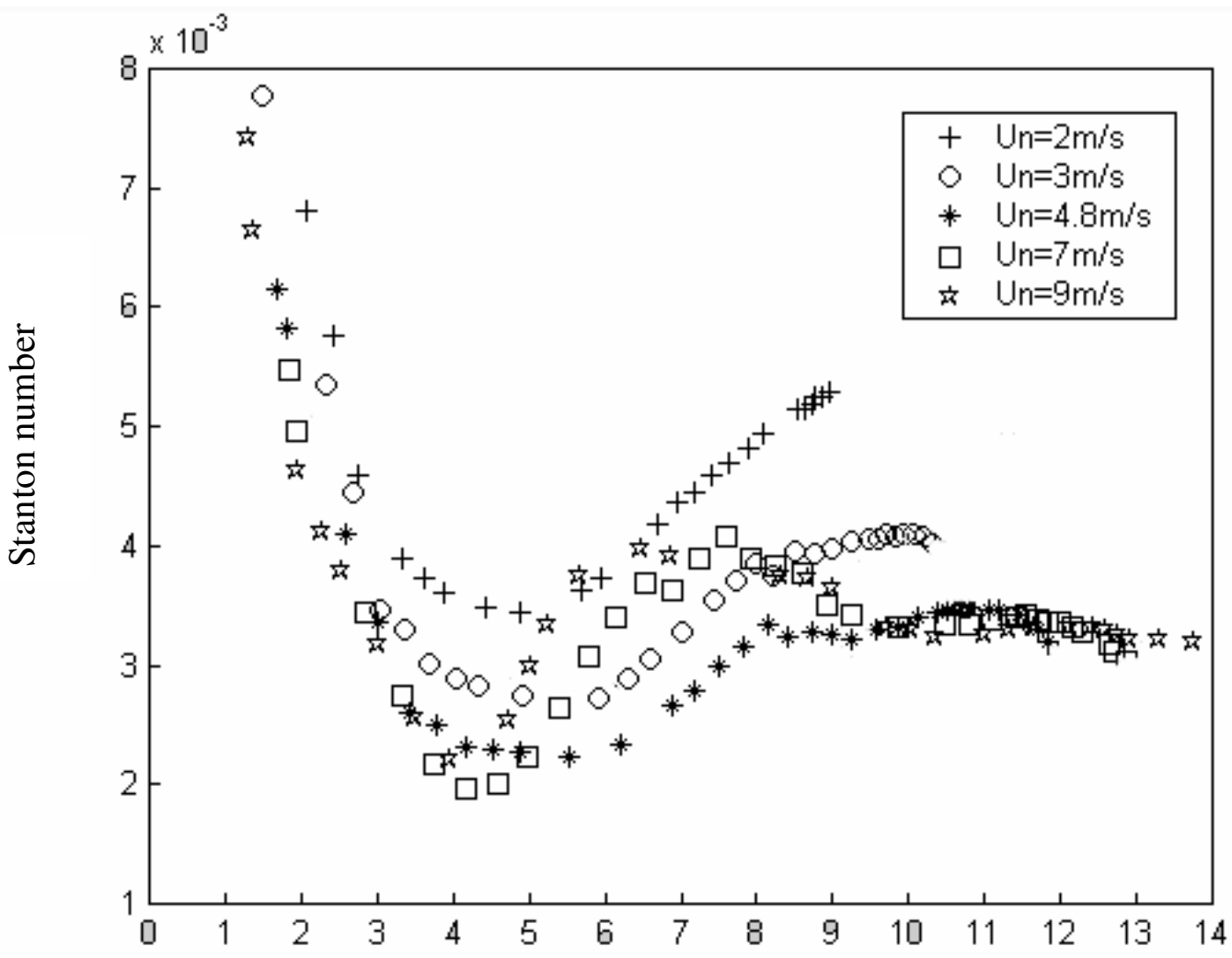

Görtler number

Figure 5. Evolution of the Stanton number as a function of Görtler number for various free-stream velocities in the absence of the vortex triggering grid. 
2

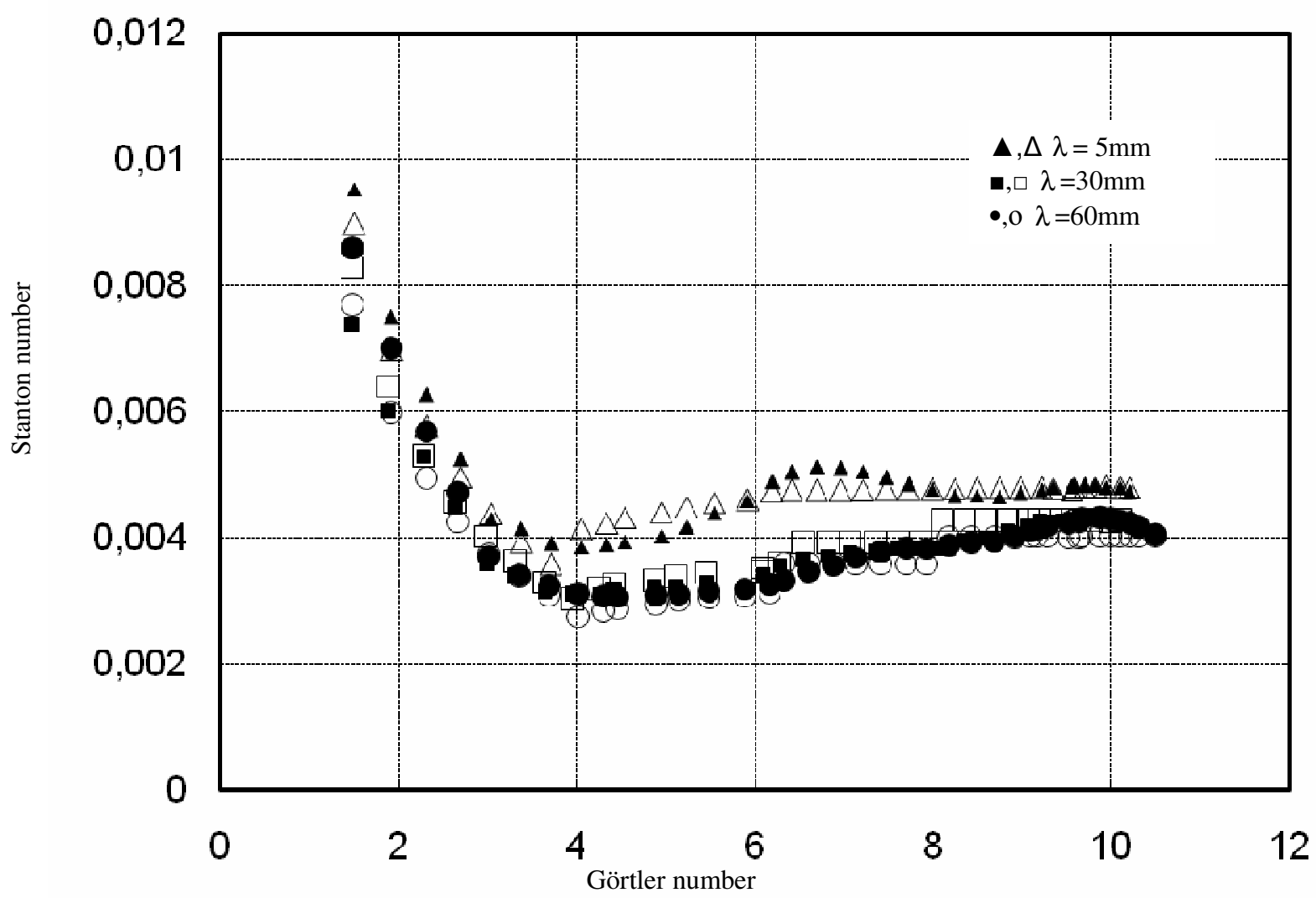

Figure 6. Comparison between experimental (filled) and simulated (empty)

evolution of Stanton number versus Görtler number for different $\lambda$ and $U_{n}=3 \mathrm{~m} / \mathrm{s}$,

$\mathrm{d}_{\mathrm{w}}=0.18 \mathrm{~mm}$. Simulation results are obtained from the second correlation. 


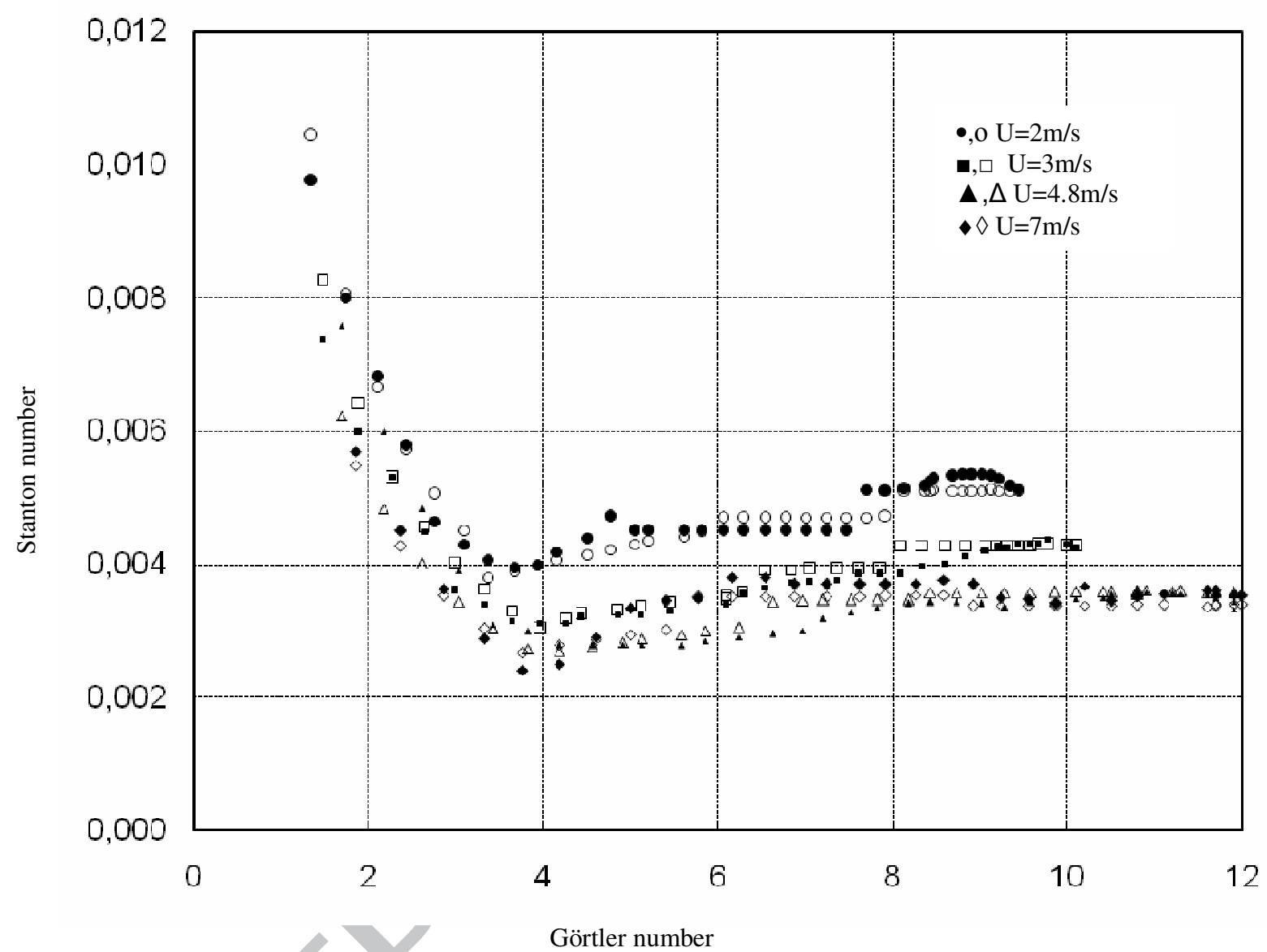

Figure 7 Comparison between experimental (filled) and simulated (empty) evolution of Stanton number versus Görtler number for different $U$ and $\lambda=30 \mathrm{~mm}$, $\mathrm{d}_{\mathrm{w}}=0.18 \mathrm{~mm}$. Simulation results are obtained from the second correlation. 


\begin{tabular}{|l|l|l|l|l|l|l|l|l|}
\hline$d_{w}(m m)$ & $a(O A)$ & $b(O A)$ & $c(O A)$ & $d(O A)$ & $a(A B)$ & $b(A B)$ & $c(A B)$ & $d(A B)$ \\
\hline 0.18 & 0.182 & -0.891 & -0.352 & -0.067 & 0.012 & -0.106 & 0.201 & -0.163 \\
\hline 0.8 & 0.208 & -0.825 & -0.341 & -0.140 & 0.023 & -0.022 & 0.036 & -0.179 \\
\hline$d_{w}(m m)$ & $a(B C)$ & $b(B C)$ & $c(B C)$ & $d(B C)$ & $a(C D)$ & $b(C D)$ & $c(C D)$ & $d(C D)$ \\
\hline 0.18 & 0.018 & -0.212 & 0.060 & -0.115 & 0.08 & -0.495 & 0.229 & -0.074 \\
\hline 0.8 & 0.083 & -0.517 & 0.149 & -0.048 & 0.100 & -0.573 & 0.198 & 0 \\
\hline
\end{tabular}

Table 3: Values of different coefficients for second correlation: $\mathrm{St}=\mathrm{a}\left(\mathrm{R}_{\theta}\right)^{\mathrm{b}}(\mathrm{x} / \mathrm{R})^{\mathrm{c}}(\lambda / \theta)^{\mathrm{d}}$. 


\begin{tabular}{|l|l|l|l|l|}
\hline $\mathrm{U}(\mathrm{m} / \mathrm{s})$ & 2 & 3 & 4.8 & 7 \\
\hline first correlation & 6.09 & 4.76 & 7.63 & 10.15 \\
\hline second correlation & 5.18 & 5.03 & 7.17 & 9.07 \\
\hline third correlation & 7.99 & 7.75 & 11.704 & 14.45 \\
\hline
\end{tabular}

(a)

\begin{tabular}{|l|l|l|l|l|}
\hline $\mathrm{U}(\mathrm{m} / \mathrm{s})$ & 2 & 3 & 4.8 & 7 \\
\hline first correlation & 2.79 & 5.57 & 6.23 & 4.86 \\
\hline second correlation & 6.23 & 6.32 & 6.64 & 6.22 \\
\hline third correlation & 6.96 & 7.10 & 10.15 & 8.02 \\
\hline
\end{tabular}

(b)

Table 4: Mean values of errors (\%) for different experimental configurations in the present work, for first correlation $S t=\mathrm{aG}_{\theta}^{\mathrm{b}} \Lambda^{\mathrm{c}} \operatorname{Re}_{\mathrm{x}}^{\mathrm{d}}$, for second correlation $S t=a\left(R_{\theta}\right)^{b}(x / R)^{c}(\lambda / \theta)^{d}$, and for third correlation $\mathrm{St}=\mathrm{a}\left(\frac{\mathrm{U}_{\mathrm{n}} \lambda}{\mathrm{v}}\right)^{\mathrm{b}}\left(\frac{\theta}{\mathrm{R}}\right)^{\mathrm{c}}$, (a) $\mathrm{d}_{\mathrm{w}}=0.18 \mathrm{~mm},(\mathrm{~b}) \mathrm{d}_{\mathrm{w}}=0.80 \mathrm{~mm}$ 


\begin{tabular}{|l|l|}
\hline$G_{\theta}$ & $1-14$ \\
\hline$U_{n}(\mathrm{~m} / \mathrm{s})$ & $2-9$ \\
\hline$\lambda(\mathrm{cm})$ & $0.5-6$ \\
\hline $\operatorname{Re}_{x}$ & $0.2 \times 10^{5}-4 \times 10^{5}$ \\
\hline $\mathrm{X}(\mathrm{cm})$ & $0-70$ \\
\hline $\mathrm{R}(\mathrm{cm})$ & 65 \\
\hline
\end{tabular}

Table 3: The range of parameters covered in the experiments and in simulations reported in this work . 